\title{
Aspects of solar wind interaction with Mars: comparison of fluid and hybrid simulations
}

\author{
N. V. Erkaev ${ }^{1}$, A. Bößwetter ${ }^{2}$, U. Motschmann ${ }^{2}$, and H. K. Biernat ${ }^{3}$ \\ ${ }^{1}$ Institute for Computational Modelling, Russian Academy of Sciences, 660036, Krasnoyarsk, Russia \\ ${ }^{2}$ Institute for Theoretical Physics, TU Braunschweig, Germany \\ ${ }^{3}$ Space Research Institute, Austrian Academy of Sciences, Graz, Austria
}

Received: 9 May 2006 - Revised: 11 October 2006 - Accepted: 10 January 2007 - Published: 1 February 2007

\begin{abstract}
Mars has no global intrinsic magnetic field, and consequently the solar wind plasma interacts directly with the planetary ionosphere. The main factors of this interaction are: thermalization of plasma after the bow shock, ion pick-up process, and the magnetic barrier effect, which results in the magnetic field enhancement in the vicinity of the obstacle. Results of ideal magnetohydrodynamic and hybrid simulations are compared in the subsolar magnetosheath region. Good agreement between the models is obtained for the magnetic field and plasma parameters just after the shock front, and also for the magnetic field profiles in the magnetosheath. Both models predict similar positions of the proton stoppage boundary, which is known as the ion composition boundary. This comparison allows one to estimate applicability of magnetohydrodynamics for Mars, and also to check the consistency of the hybrid model with Rankine-Hugoniot conditions at the bow shock. An additional effect existing only in the hybrid model is a diffusive penetration of the magnetic field inside the ionosphere. Collisions between ions and neutrals are analyzed as a possible physical reason for the magnetic diffusion seen in the hybrid simulations.
\end{abstract}

Keywords. Interplanetary physics (Planetary bow shocks; Solar wind plasma) - Space plasma physics (Numerical simulation studies)

\section{Introduction}

Mars does not have a sufficient magnetic field to form its own magnetosphere, and thus the solar wind interacts directly with the ionosphere of the planet. Even though the planet is unmagnetized, the flow of the solar wind is strongly affected by the interplanetary magnetic field (IMF) which plays a crucial role in the interaction of the solar wind pro-

Correspondence to: N. V. Erkaev

(erkaev@icm.krasn.ru) tons with the ionospheric plasma. The magnetic field has a jump at the bow shock and then it becomes much stronger as the ionopause is approached, giving rise to the magnetic barrier with a distinct magnetic pile-up boundary (MPB), which is identified with the magnetic field rotation and the drop in the magnetic turbulence level. This boundary was detected by FGMM (Sauer et al., 1990), as well as by the Phobos-2 MAGMA instrument (Riedler et al., 1991), and also by MGS (Vignes et al., 2000). The magnetic pressure maximum is of the order of the solar wind dynamic pressure, and thus the magnetic field strength at the MPB is proportional to the solar wind bulk speed. As shown by Biernat et al. (1999), the magnetic field profiles across the magnetosheath become steeper when the solar wind Alfvén Mach number increases.

A peculiarity of the solar wind interaction with the unmagnetized planets is that the neutral atmospheric atoms can be ionized and involved into the solar wind flow. This is called the mass loading process characterized by the ionization rate depending on the solar activity. The loaded heavy ions form a dense layer with a so-called "ion composition boundary" (ICB) separating the solar wind protons from the planetary ions. This boundary was identified by ion measurements from the ASPERA and TAUS experiments on board Phobos-2 (Rosenbauer et al., 1989; Breus et al., 1991; Sauer et al., 1994). Analysis of the multi-instrument observations (see Nagy et al., 2004, and references therein) clearly indicates that the drop in the proton density and the pile-up of the electron density at the ICB typically coincide with the MPB position.

Analysis of observations and simulations highlights two aspects of the magnetic field enhancement caused by the solar wind interaction with Mars. The first one is the magnetic barrier in the subsolar magnetosheath, where the magnetic field strength is gradually increasing from the bow shock to the proton cavity boundary. This effect exists even without mass loading process and was still obtained in the gasdynamic model of Spreiter and Stahara (1980), which

Published by Copernicus GmbH on behalf of the European Geosciences Union. 

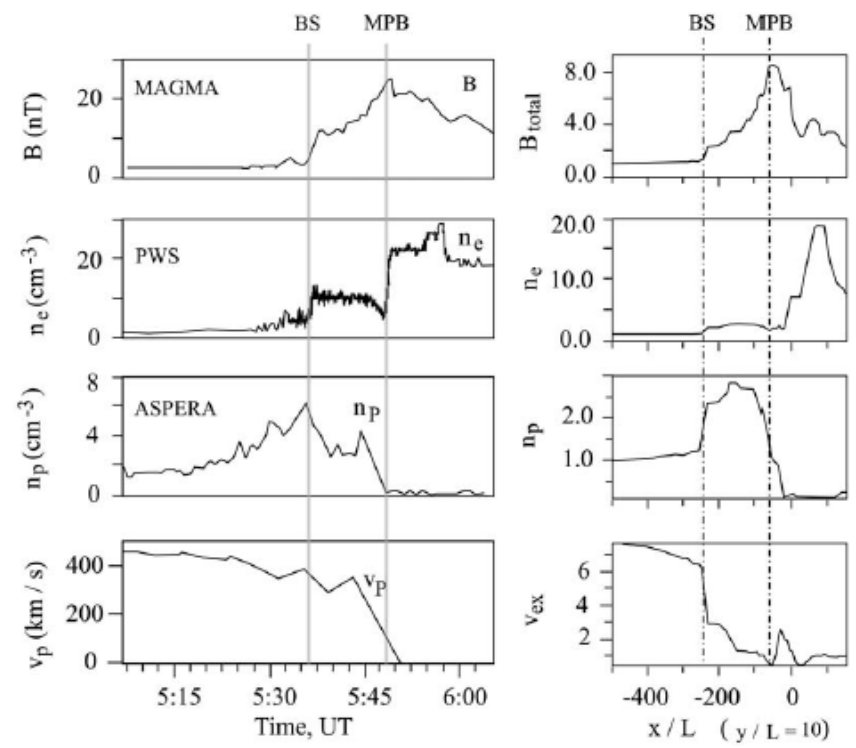

Fig. 1a. Comparison between Phobos-2 measurements (left panels) and bi-ion fluid simulations (right panels) for the third elliptical orbit, after Sauer and Dubinin (2000).

overestimates the magnetic field strength due to a kinematic treatment of the frozen-in magnetic field lines. It was also introduced by Zwan and Wolf (1976) as a plasma depletion layer for the Earth's magnetosheath. This effect can be reproduced by a one-fluid magnetohydrodynamic (MHD) model, and also by multi-fluid MHD and hybrid models. However, the effect might be weakened by the magnetic diffusion related to a numerical procedure. Such magnetic field enhancement in the magnetosheath can be seen in Fig. 1a, which demonstrates Phobos-2 measurements at the left panels, and bi-ion fluid simulations at the right panels for the third elliptical orbit, after Sauer and Dubinin (2000). In this figure, the magnetic field strength is gradually increasing in the magnetosheath from the bow shock towards the ICB/MPB, where it reaches a maximum. The total density first has a jump at the bow shock, then it decreases towards the ICB/MPB, where it has a pile-up.

The second aspect is the formation of the induced magnetosphere inside the proton cavity separated from the magnetosheath by ICB/MPB. This is not reproduced by the ideal MHD model, but in principle, it can be described by bi-ion and hybrid models.

The applicability of the MHD model for the solar wind interaction with Mars is questionable because the kinetic plasma scales (Larmour radius, ion inertial scale) are not significantly less than the magnetosheath scales. In particular, the Larmour radius of the cold heavy ions accelerated by the electric field can be of the same order of magnitude as the magnetic barrier length scale. Nevertheless, the previous magnetohydrodynamic (MHD) simulations by Liu et

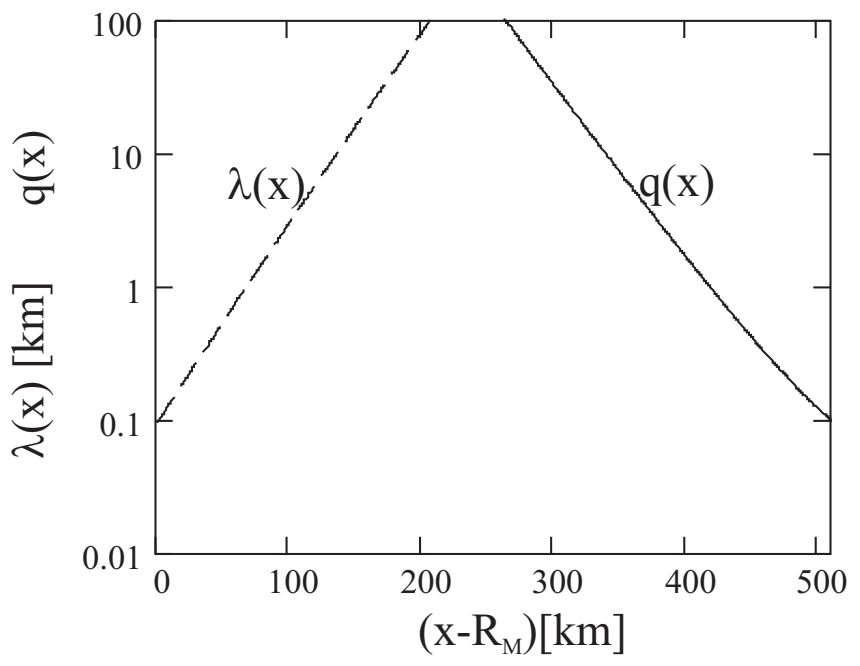

Fig. 1b. Collision free path $\lambda(x)$ (dash line), and the dimensionless mass loading parameter $q(x)$ (solid line).

al. (2001) and Ma et al. (2004) showed the positions of the bow shock and the ionopause to be in good agreement with observations. However, MPB/ICB was not reproduced well. In a two-fluid MHD model, Sauer and Dubinin (2000) revealed that the mass loading of the ions may result in a sudden stoppage of the proton flow and the formation of the proton cavity around the ionopause. The cavity boundary is associated with the ICB/MPB.

Semi-kinetic simulations often used in space research are the so-called hybrid simulations, where the ions are treated as individual particles, and the electrons are considered to be a fluid. This approach has been applied by Brecht et al. (1993) for simulations of the solar wind interaction with Mars. A comparison of the simulation results by Brecht (1997) with the data obtained by spacecraft Phobos 2 yields a consistency between the resultant large-scale magnetic field configuration around Mars and the observations.

In the hybrid simulation by Shimazu (2001), the planet was treated as a gaseous body, which could be penetrated by the ionospheric plasma, produced with a constant ionization rate. The simulation predicted an asymmetric bow shock with a multiple-shock structure, and also a magnetic barrier in front of the planet with asymmetries along the solar wind electric field. Kallio and Janhunen (2001) applied a hybrid model to study the atmospheric effects of proton precipitation in the atmosphere and the ion escape at the nightside of the planet (Kallio and Janhunen, 2002). The simulations showed a draping of the magnetic field lines around the planet, but the plasma boundaries were not reproduced clearly. Kallio and Janhunen (2002) do not include an electron pressure term at all, whereas Shimazu (2001) uses a global electron pressure gradient for the solar wind plasma and the planetary ions plasma. The aspects of 
ICB/MPB were studied in hybrid simulations by Bößwetter et al. (2004), which are based on a more detailed model including two electron fluids with different temperatures, as well as a friction force between ions and neutral gas atoms.

The MPB and ICB are nearly the same in the observations, but they describe a behavior of the different physical quantities: magnetic field and plasma density. In the model simulations, these boundaries are not completely identical. Therefore, we refer to both terms, ICB and MPB, which are reproduced differently in model simulations. In particular, MHD and hybrid simulations can give very distinct ICB, as a sudden rise in the electron density and decrease in the proton density. However, the variations of the magnetic field components through this boundary are smoothed by the numerical diffusion.

Applications of complicated models (multi-fluid and hybrid) allow one to obtain a lot of information about the object of simulation, but require to reduce the resolution. There also exists a problem in distinguishing the physical results from the numerical ones. In particular, this concerns the numerical magnetic diffusion effects existing in global numerical simulations. Therefore, for clarifying the physical processes on the magnetosheath flow it is useful to compare relevant results obtained in frameworks of different simulation approaches. Comparison of different models provides a better understanding of the physical meaning of simulation results.

The aim of this paper is to compare the simulation results for the magnetosheath obtained from the MHD and hybrid models. The main scope of our study concerns the dayside magnetosheath profiles of the magnetic field and plasma parameters from the bow shock to the ICB/MPB. This comparison is useful for a better estimation of the potential and limits of the MHD model and its relationship with the kinetic model. An additional aspect to be clarified is the physical reason for the magnetic field diffusion inside the dayside Martian ionosphere, which can be seen in the hybrid simulations.

\section{Basic equations and input parameters}

We model the plasma in the planetary magnetosheath as a nondissipative fluid which obeys the ideal MHD equation (in cgs units) based on the conservation laws for momentum, mass, energy and also on the frozen-in condition for the magnetic field,

$$
\begin{aligned}
& \nabla \cdot\left[\rho \boldsymbol{u} \boldsymbol{u}+\Pi \mathbf{I}-\frac{1}{4 \pi} \boldsymbol{B} \boldsymbol{B}\right]=0, \\
& \nabla \cdot(\rho \boldsymbol{u})=Q, \quad \nabla \cdot \boldsymbol{B}=0, \quad \nabla \times(\boldsymbol{u} \times \boldsymbol{B})=0, \\
& \nabla \cdot\left[\boldsymbol{u}\left(\rho \frac{u^{2}}{2}+\frac{\kappa}{\kappa-1} P+\frac{1}{4 \pi} B^{2}\right)-\right. \\
& \left.\frac{1}{4 \pi} \boldsymbol{B}(\boldsymbol{u} \cdot \boldsymbol{B})\right]=0 .
\end{aligned}
$$

In Eq. (1), $\Pi$ is the sum of the gas and magnetic pressures, $\Pi=P+B^{2} / 8 \pi$; quantities $P$ and $\rho$ are the gas pressure and the mass density, respectively, and $\boldsymbol{B}$ and $\boldsymbol{u}$ are the magnetic field and the plasma flow velocity, respectively, parameter " $\kappa$ " denotes the ratio of specific heats $(\kappa=5 / 3), Q$ is a source function determined by the ionization rate and the distribution of the neutral particles,

$Q=M_{i} N_{0}(r) v f(r, \chi)$,

where $M_{i}$ is the mass of the heavy ions (oxygen), $v$ is the ionization rate, $N_{0}$ is the density of neutral particles depending on the radius, $f(r, \chi)$ is a Chapman-like function of the radial distance and the solar zenith angle (Chamberlain and Hunten, 1987). We use the distribution of the neutral density and function $f(r, \chi)$ similar to those suggested by Bößwetter et al. (2004),

$$
\begin{aligned}
& N_{0}(r)=n_{1} \exp \left(\frac{r_{1}+R_{M}-r}{H_{1}}\right)+ \\
& n_{2} \exp \left(\frac{r_{2}+R_{M}-r}{H_{2}}\right)+n_{3} \frac{r_{3}}{\left(r-R_{M}\right)}, \\
& f(r, \chi)=\exp \left[-\frac{\sigma n_{1} H_{1}}{\cos (\chi)}\left[\exp \left(\frac{r_{1}+R_{M}-r}{H_{1}}\right)+\right.\right. \\
& \frac{n_{2} H_{2}}{n_{1} H_{1}} \exp \left(\frac{r_{2}+R_{M}-r}{H_{2}}\right)+ \\
& \left.\left.\frac{n_{3} r_{3}}{n_{1} H_{1}} \log \left(\frac{R_{M}}{r-R_{M}}\right)\right]\right],
\end{aligned}
$$

where $R_{M}$ is the planet's radius, $R_{M}=3400 \mathrm{~km}$; parameters $n_{1}, n_{2}, n_{3}, r_{1}, r_{2}, r_{3}$ are chosen to fit the data given by Chen et al. (1978), Kallio and Luhmann (1997) and Kotova et al. (1997) for solar minimum conditions. The resulting values are $n_{1}=2.8 \times 10^{14} \mathrm{~m}^{-3}, n_{2}=2.5 \times 10^{12} \mathrm{~m}^{-3}, n_{3}=10^{9} \mathrm{~m}^{-3}$, $r_{3}=1700 \mathrm{~km}, r_{1}=140 \mathrm{~km}, r_{2}=300 \mathrm{~km}, H_{1}=27 \mathrm{~km}, H_{2}=35 \mathrm{~km}$. Boundary conditions are given at the bow shock (RankineHugoniot) and at the ionopause $u_{n}=0$.

The initial conservative system of MHD Eqs. (1-3) can be transformed to the dimensionless system which is more suitable for computations,

$$
\begin{aligned}
& \rho(\boldsymbol{u} \cdot \nabla) \boldsymbol{u}+\nabla \Pi=\frac{1}{M_{A}^{2}}(\boldsymbol{B} \cdot \nabla) \boldsymbol{B}-q(\boldsymbol{r}) \boldsymbol{u}, \\
& \nabla \cdot(\rho \boldsymbol{u})=q(\boldsymbol{r}), \quad \nabla \cdot \boldsymbol{B}=0, \quad \nabla \times(\boldsymbol{u} \times \boldsymbol{B})=0, \\
& (\boldsymbol{u} \cdot \nabla)\left(\frac{P}{\rho^{\kappa}}\right)=\frac{Q}{\rho^{\kappa+1}} \frac{(\kappa-1)}{2}\left[\rho u^{2}-\frac{P \kappa}{(\kappa-1)}\right] .
\end{aligned}
$$

Here velocity $\boldsymbol{u}$, magnetic field $\boldsymbol{B}$ and density $\rho$ are normalized to their solar wind values $u_{s w}, B_{s w}, \rho_{s w}$, respectively; plasma pressure is normalized to the solar wind dynamic pressure $\rho_{s w} u_{s w}^{2}$, distances are normalized to the planetary radius $R_{M} ; M_{A}$ is a solar wind Alfvén Mach number, $M_{A}=\sqrt{4 \pi \rho_{s w}} u_{s w} / B_{s w} ; q$ is the dimensionless mass loading function characterizing the efficiency of the ion pick-up process,

$q(\boldsymbol{r})=Q(\boldsymbol{r}) \frac{R_{M}}{\rho_{s w} u_{s w}}$. 
Mass loading effects are strongly pronounced in the region where $q(\boldsymbol{r}) \gg 1$.

Equation (7) contains the additional resistance force proportional to the velocity, which is related to the mass loading process. This force leads to the deceleration of the protons which lose energy for the corresponding acceleration of the heavy ions. Equation (9) indicates that the adiabatic law is not valid anymore due to the mass loading effect.

The collision length scale related to the interaction with the neutral particles is defined as $\lambda=V^{*} / K_{D} N_{0}$, where $V^{*}$ $=10 \mathrm{~km} / \mathrm{s}$, constant $K_{D}=1.7 \times 10^{-9} \mathrm{~cm}^{3} \mathrm{~s}^{-1}$ is given by Israelevich et al. (1999). This constant $K_{D}$ determines the collisional forces $\boldsymbol{F}_{c i}=-K_{D} N_{0} M_{i}\left(\boldsymbol{u}_{i}-\boldsymbol{u}_{n}\right)$ between the ions and ionospheric neutrals, which were used in the hybrid simulations by Bößwetter et al. (2004). The characteristic velocity $V^{*}$ is chosen to be of the order of the proton's velocity near the ICB obtained in the hybrid simulations by Bößwetter et al. (2004).

Figure $1 \mathrm{~b}$ shows the collision length scale $\lambda(x)$ and also the dimensionless mass loading parameter $q(x)$ as functions of the distance along the $\mathrm{x}$-axis. Assuming the condition for the collision zone $\lambda(x)<50 \mathrm{~km}$ we find the altitude of about $0.05 R_{m}=170 \mathrm{~km}$.

As shown by Hanson and Mantas (1988), the ionospheric peak of the thermal pressure at Mars is smaller than the average solar wind dynamic pressure. In such a case, at the subsolar region, the solar wind protons can penetrate into the collision zone.

For the MHD simulation, the effective obstacle is disposed in front of the subsolar collision zone. The model shape of the obstacle is the axisymmetrical surface consisting of a hemisphere on the sunward side, smoothly attached to a slightly expanding surface on the night side.

The input solar wind parameters used in our calculations are

$$
\begin{aligned}
n_{s w} & =4 \mathrm{~cm}^{-3}, \quad u_{s w}=327 \mathrm{~km} / \mathrm{s}, \quad B_{s w}=3 \mathrm{nT}, \\
T_{e, s w} & =2 \times 10^{5} \mathrm{~K}, \quad T_{p, s w}=0.5 \times 10^{5} \mathrm{~K} .
\end{aligned}
$$

This upstream parameters represent reasonable values. In particular, IMF and the total ram pressure, as well as the Alfvén Mach number are nearly identical to those corresponding to Phobos-2's elliptical orbit on 8 February 1989 (see Sauer and Dubinin, 2000).

The supersonic solar wind flow is decelerated and thermalized at the detached bow shock, where the Rankine-Hugoniot conditions have to be fulfilled. At the obstacle the normal component of bulk velocity is assumed to vanish.

\section{Numerical technique for MHD model}

The numerical/analytical technique we use was previously applied by Erkaev et al. (1996, 1998, 1999, 2003) for the solar wind flow around the magnetospheres of Jupiter and
Earth, and also by Biernat et al. $(1999,2001)$ for the solar wind interaction with the ionosphere of Venus.

To describe an ideally conducting plasma, we use the material frozen-in coordinates $(\alpha, \lambda, \tau)$, which are similar to those introduced by Pudovkin and Semenov (1977). For a stationary flow, these coordinates can be introduced by the equations: $\boldsymbol{u} \cdot \nabla \alpha=0, \boldsymbol{u} \cdot \nabla \lambda=0, \boldsymbol{u} \cdot \nabla \tau=1$. A particular pair of $(\alpha, \lambda)$ labels a streamline, and the coordinate $\tau$ has the sense of time of motion of a fluid particle along its streamline. These coordinates are specified to be proportional to Cartesian coordinates in the unperturbed, upstream flow. In this case, variables $(\lambda, \tau)$ are constant along the magnetic field lines everywhere. In frozen-in coordinates, the nondissipative MHD equations can be written as follows,

$$
\begin{aligned}
& \frac{\partial \boldsymbol{u}}{\partial \tau}-\frac{J}{M_{A}^{2}} \frac{\partial \boldsymbol{B}}{\partial \alpha}+\frac{1}{\rho} \nabla \Pi(\boldsymbol{r})=-\frac{q}{\rho} \boldsymbol{u}, \\
& \frac{\partial}{\partial \tau}\left(\frac{\boldsymbol{B}}{J \rho}\right)-\frac{\partial \boldsymbol{u}}{\partial \alpha}=0, \quad \frac{\partial \boldsymbol{r}}{\partial \tau}=\boldsymbol{u}, \\
& \frac{\partial J}{\partial \tau}=-\frac{q}{\rho} J, \quad S \rho^{\kappa}+\frac{B^{2}}{2 M_{A}^{2}}=\Pi(\boldsymbol{r}), \\
& \frac{\partial S}{\partial \tau}=\frac{q}{2 \rho^{\kappa}}\left[(\kappa-1) u^{2}-\kappa S \rho^{\kappa-1}\right], \\
& \frac{D(x, y, z)}{D(\alpha, \lambda, \tau)}=\frac{1}{J \rho} .
\end{aligned}
$$

Here $D(..) / D(.$.$) denotes the Jacobian of the transformation,$ $J$ is the ratio of the proton and total mass densities, $\boldsymbol{r}$ is the position vector normalized to the radius of the obstacle. Vector function $\boldsymbol{r}(\alpha, \lambda, \tau)$ describes the positions of the frozenin magnetic field lines characterized by constant values of two material coordinates, $\lambda$ and $\tau$. The third coordinate $\alpha$ varies from $-\infty$ to $+\infty$ along each field line. Our method was developed from the boundary layer approach based on approximations for the total pressure in the magnetosheath. Initially, we assume a quadratic variation of the total pressure between the bow shock and the obstacle. At the bow shock it is determined from the Rankine-Hugoniot conditions, and at the obstacle it is given by the Newtonian formula that is well known in the gasdynamics as a good analytical approximation for the pressure on a streamlined obstacle (Petrinec and Russell, 1997). The bow shock is approximated initially as a hyperboloid of revolution. The total pressure behavior and the bow shock shape are corrected at the next iterations. We consider the tangential and the normal components (with respect to the obstacle) of the momentum and induction Eqs. (12) and (13). Using a finite difference Lax-Wendroff scheme, we integrate the tangential momentum and induction equations, together with Eqs. (14) and (15) from the bow shock to the obstacle, and thus we obtain the tangential components of the magnetic field and velocity and also the mass density as functions of the material coordinates. The normal components of the magnetic field and velocity, as well as a normal displacement of the magnetic field lines are obtained 
by integration of the Jacobian Eq. (16) by the method of characteristics using the boundary conditions at the obstacle. This yields a correction of the bow shock position. After that we correct the total pressure distribution along the normal directions using the normal momentum equation, as well as the Rankine-Hugoniot conditions at the bow shock. Using the corrected total pressure distribution and the updated bow shock position, we repeat the calculations for the next iteration. This approach based on the frozen-in coordinates allows us to avoid the effects of numerical magnetic diffusion.

\section{Description of hybrid model}

The numerical hybrid simulations are performed using a code described by Bößwetter et al. (2004). In the hybrid approximation the electrons are modelled as a massless charge neutralizing fluid, whereas the ions are treated as individual particles. A collisional force between the ions and the ionospheric neutral gas atoms (neutral drag force) is included to take into account possible collisional effects. For the ions, the equation of motion is

$\frac{d \boldsymbol{u}_{i / p}}{d t}=\frac{e}{M_{i / p}}\left(\boldsymbol{E}+\frac{1}{c} \boldsymbol{u}_{i / p} \times \boldsymbol{B}\right)-K_{D} N_{0}\left(\boldsymbol{u}_{i / p}-\boldsymbol{u}_{0}\right)$,

where $M_{i / p}, \boldsymbol{u}_{i / p}$ are the mass and velocity of an individual particle (heavy ion or proton), respectively; $N_{0}$ and $u_{0}$ are the number density and bulk velocity of the neutrals; $K_{D}$ is a constant describing the collisions of the ions and neutrals, given as $1.7 \times 10^{-9} \mathrm{~cm}^{3} / \mathrm{s}$ by Israelevich et al. (1999). From the momentum conservation of the electron fluid, the electric field can be derived as

$\boldsymbol{E}=-\frac{1}{c} \boldsymbol{u}_{e} \times \boldsymbol{B}-\frac{1}{n e}\left(\nabla P_{e, s w}+\nabla P_{e, h i}\right)$,

where $\boldsymbol{u}_{e}$ denotes the electron bulk velocity, computed from the ionic currents and the overall currents by means of

$\boldsymbol{u}_{e}=\frac{\boldsymbol{J}_{i}}{e n}-\frac{c}{4 \pi n e} \nabla \times \boldsymbol{B}$.

Two different electron pressure terms in Eq. (18) are used to take into account the different electron temperatures of the solar wind and ionospheric electrons, respectively. Both electron populations are assumed to be adiabatic with two different initial temperatures, $T_{e, s w}$ and $T_{e, h i}$, for the solar wind and ionospheric electrons, respectively.

$P_{e, s w / h i}=n_{s w} k T_{e, s w / h i}\left(\frac{n_{e, s w / h i}}{n_{s w}}\right)^{\kappa}$.

The electron adiabatic exponent was assumed to be $\kappa=2$. The main unknown parameter is the ionospheric temperature for the ionospheric electron pressure. In this hybrid run we used $T_{e, h i}=3000 \mathrm{~K}$.

The time evolution of the magnetic field is determined by the induction equation,

$$
\frac{\partial \boldsymbol{B}}{\partial t}=\nabla \times\left(\boldsymbol{u}_{e} \times \boldsymbol{B}\right) \text {. }
$$

The simulation box has three spatial dimensions, with the undisturbed solar wind flowing in a positive x-direction, and the undisturbed interplanetary magnetic field being oriented perpendicular in a positive $y$-direction. The convective electric field points therefore to the negative $\mathrm{z}$-direction.

A highest resolution of about $100 \mathrm{~km}$ cells are found near the surface. The cells outside the planet never become larger than $170 \mathrm{~km}$. At the left-hand side of the simulation box, the solar wind comes in (in-flow boundary), whereas at the righthand side it leaves the box (outflow boundary). For the remaining sides some sort of free or absorbing boundary is desirable, however, that turned out to make the simulations unstable. We therefore use in-flow boundary conditions, which simply keep the field values constant. All particles, whether planetary ions or solar wind protons, are deleted when hitting the Martian surface. Consequently, there are no ion currents inside of Mars. The simulation starts without any magnetic or electric fields in the solid body. During the simulation, a penetration of the magnetic field through the ionosphere into the solid is allowed. The magnetic diffusion is accompanied by the penetration of the electric field. Thus, the interior of Mars is not completely field free. To avoid a collapse of the ionosphere through the inner boundary (surface), the solid body is approached by an homogeneous heavy ion density without any movement of the ions.

\section{Results}

The calculated magnetosheath profiles corresponding to the ideal MHD model are shown in Fig. 2 (solid lines) along the subsolar line for $y=0$ and $z=0$, and also in Fig. $3 a$ and Fig. $3 b$ in the two orthogonal planes: $x y$ (for $y=500 \mathrm{~km}$ and $z=0$ ) and $x z$ (for $z=500 \mathrm{~km}$ and $y=0$ ), respectively. These figures present variations of the total and proton densities, plasma pressure, electric field, velocity, magnetic field, magnetic pressure, and temperature as functions of the $\mathrm{x}$-coordinate. The direction of the IMF is assumed to be perpendicular to the solar wind velocity. All quantities are normalized to their values in the solar wind. The distances are given in units of $R_{M}$. At the same figures, the profiles obtained from the hybrid simulations are also presented for three simulation times. The hybrid simulation has a larger width of the magnetosheath than that obtained from the MHD model; partially, this is related to a finite thickness of the shock front which is of the order of the proton's Larmour radius $R_{B}$ that is equal to $340 \mathrm{~km}$ for the magnetic field just after the shock. However, the figures demonstrate good agreement between the MHD and the average hybrid simulation results for the total and proton densities, magnetic and plasma pressures, velocity, magnetic field and temperature just after the shock. This means that the hybrid model basically reproduces the Rankine-Hugoniot conditions at the bow shock. In both models, the proton pressure and the proton density rise at the bow shock and then they decrease towards the obstacle. One can 

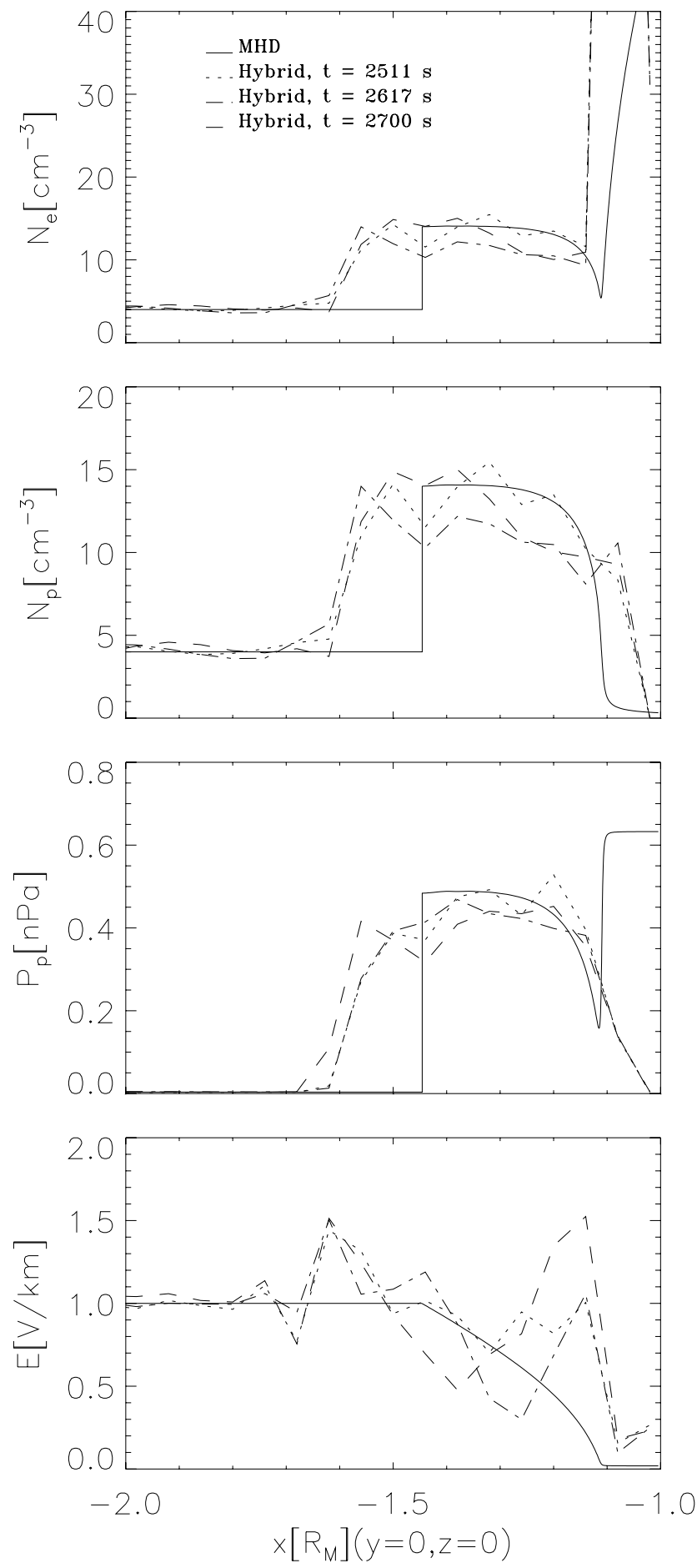
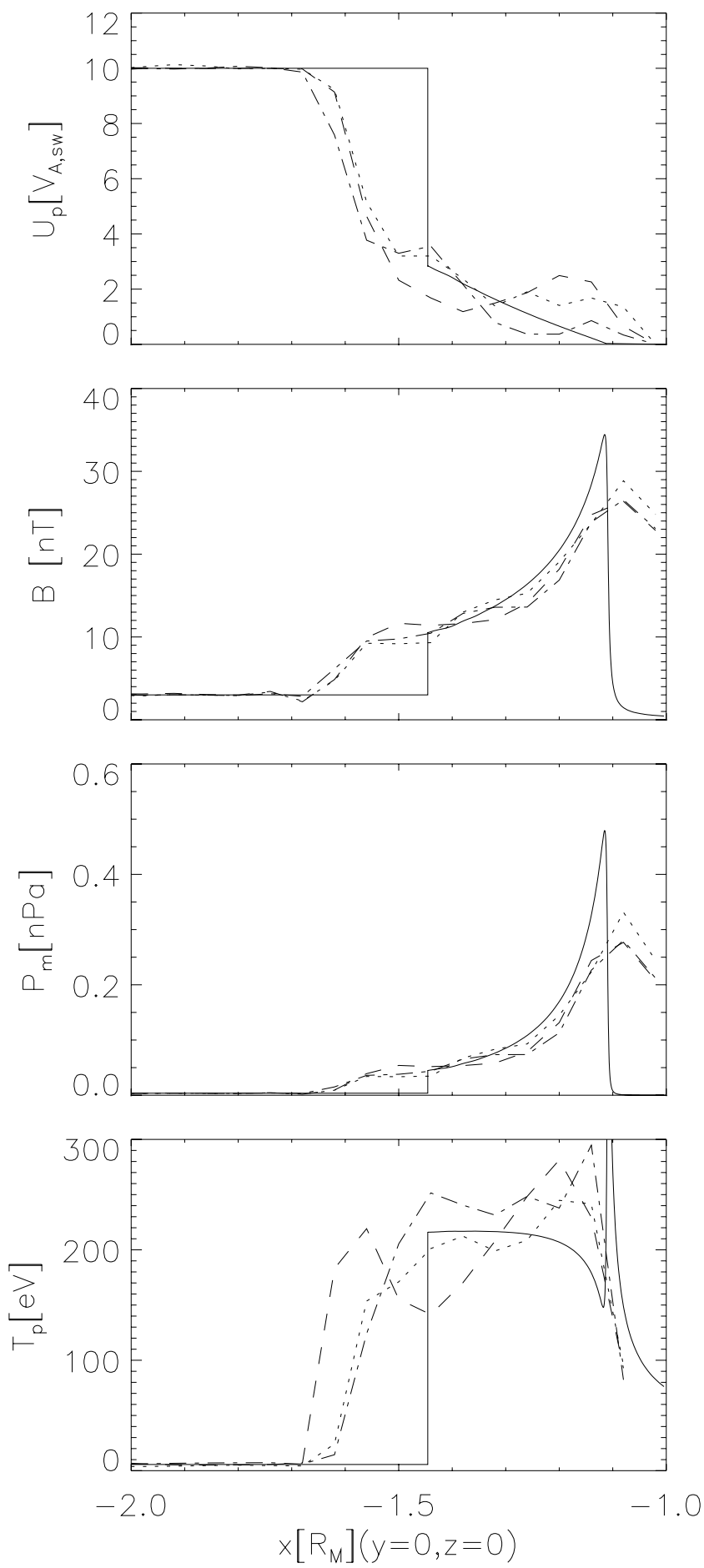

Fig. 2. Comparison of MHD and hybrid magnetosheath profiles along the subsolar line.

see in the figures that the total density has the same behavior as the proton density until some boundary where it has a very steep rise because of the appearance of the ionospheric ions that were already picked up. This pile-up of the total density seen in the figures is an indication of the ICB.
The average subsolar planetocentric distance of ICB/MPB was found to be about $1.2 R_{M}$ by Riedler et al. (1991) and $1.3 R_{M}$ by Vignes et al. (2000). However, in our simulation it is less $\left(0.1 R_{M}\right)$. A position of the ICB/MPB is very sensitive to the ionization rate. So, one reason of a low ICB/MPB 

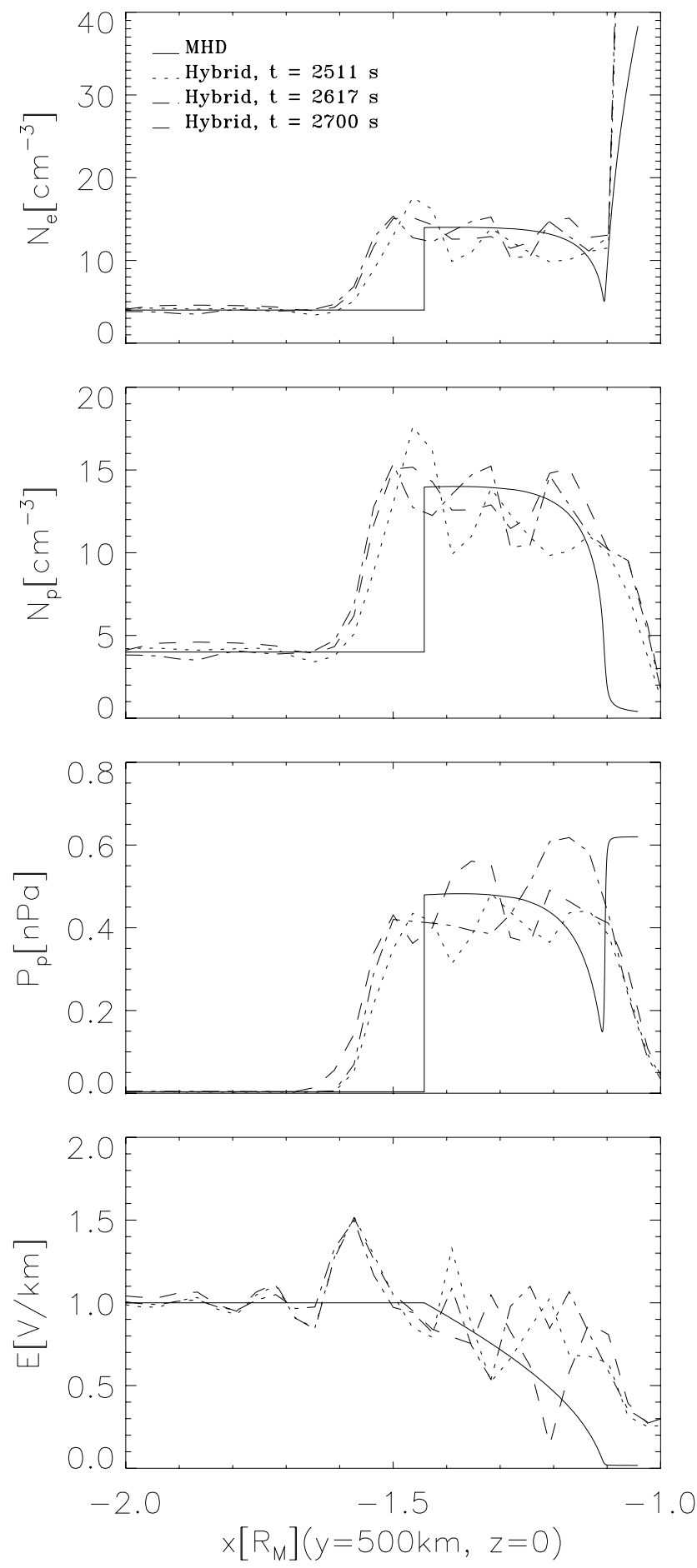
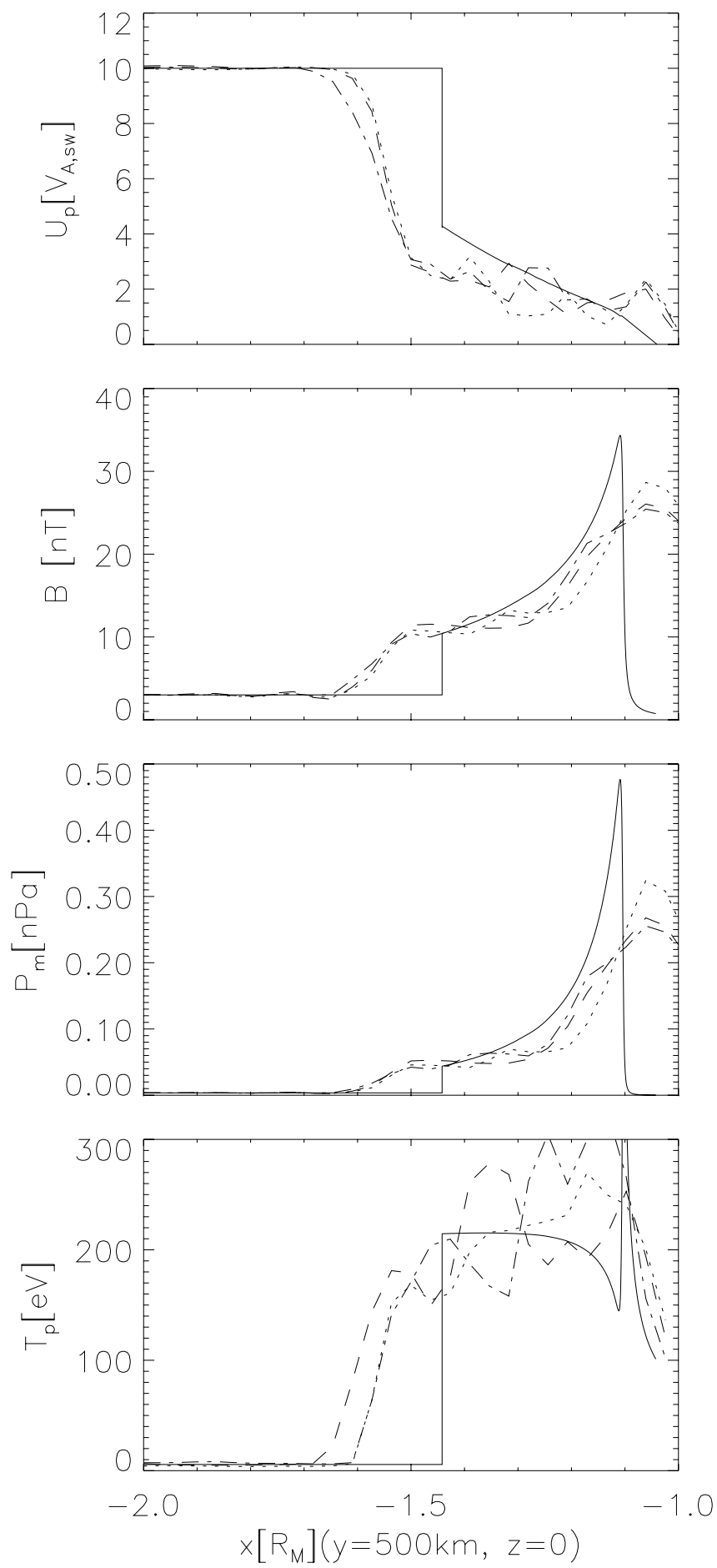

Fig. 3a. Comparison of MHD and hybrid magnetosheath profiles along the $\mathrm{x}$-direction for $y=500 \mathrm{~km}, z=0$.

distance could be related to the insufficient effective ionization rate which is the input parameter of our models. Another reason is that related to the ionospheric electron temperature is $T_{e, h}$, which is not a well known input parameter in the hybrid simulation. In the hybrid run we used $T_{e, h}=3000 \mathrm{~K}$. A choice of larger values of $T_{e, h}$ will lead certainly to higher MPB positions.

In the MHD model, a stand-off distance of the bow shock for a fixed Alfvén Mach number is proportional to the effective obstacle radius. For MGS data, the average bow shock 

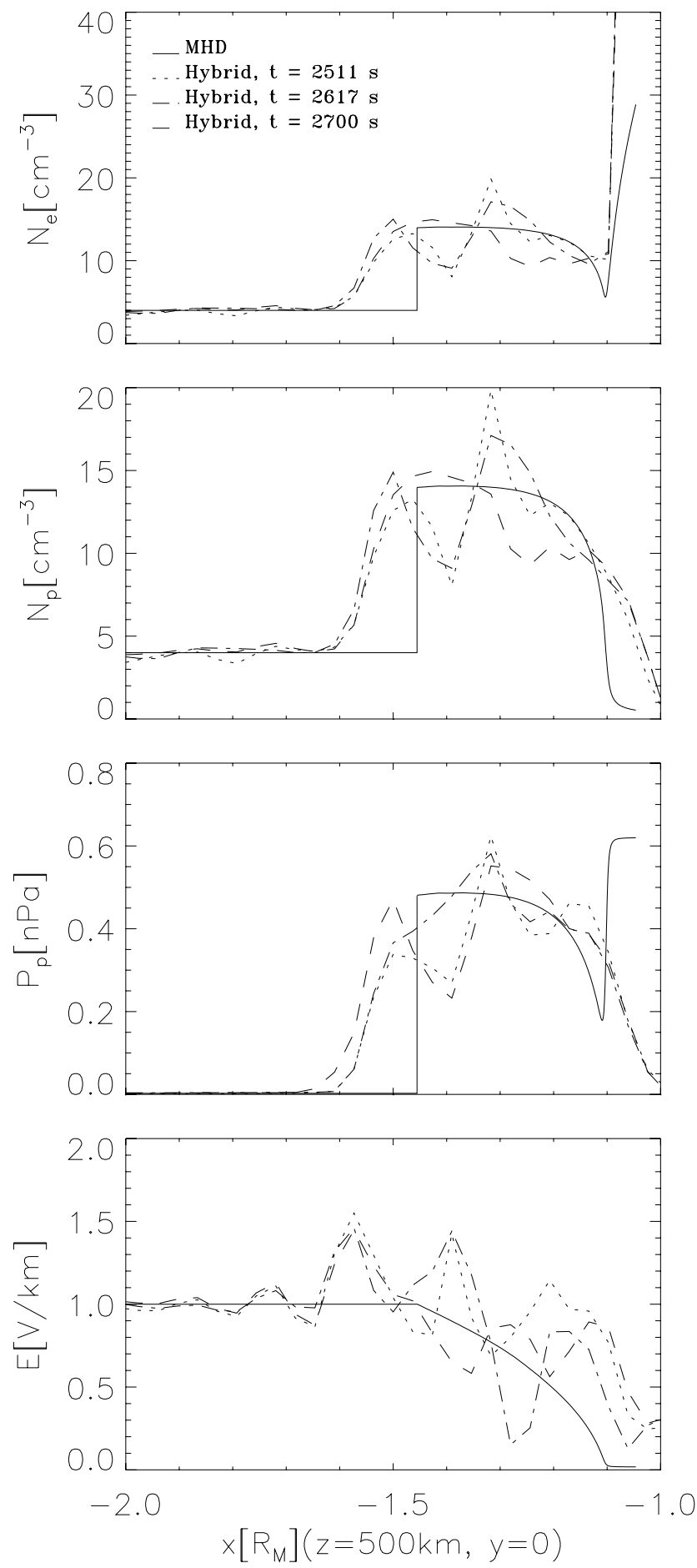
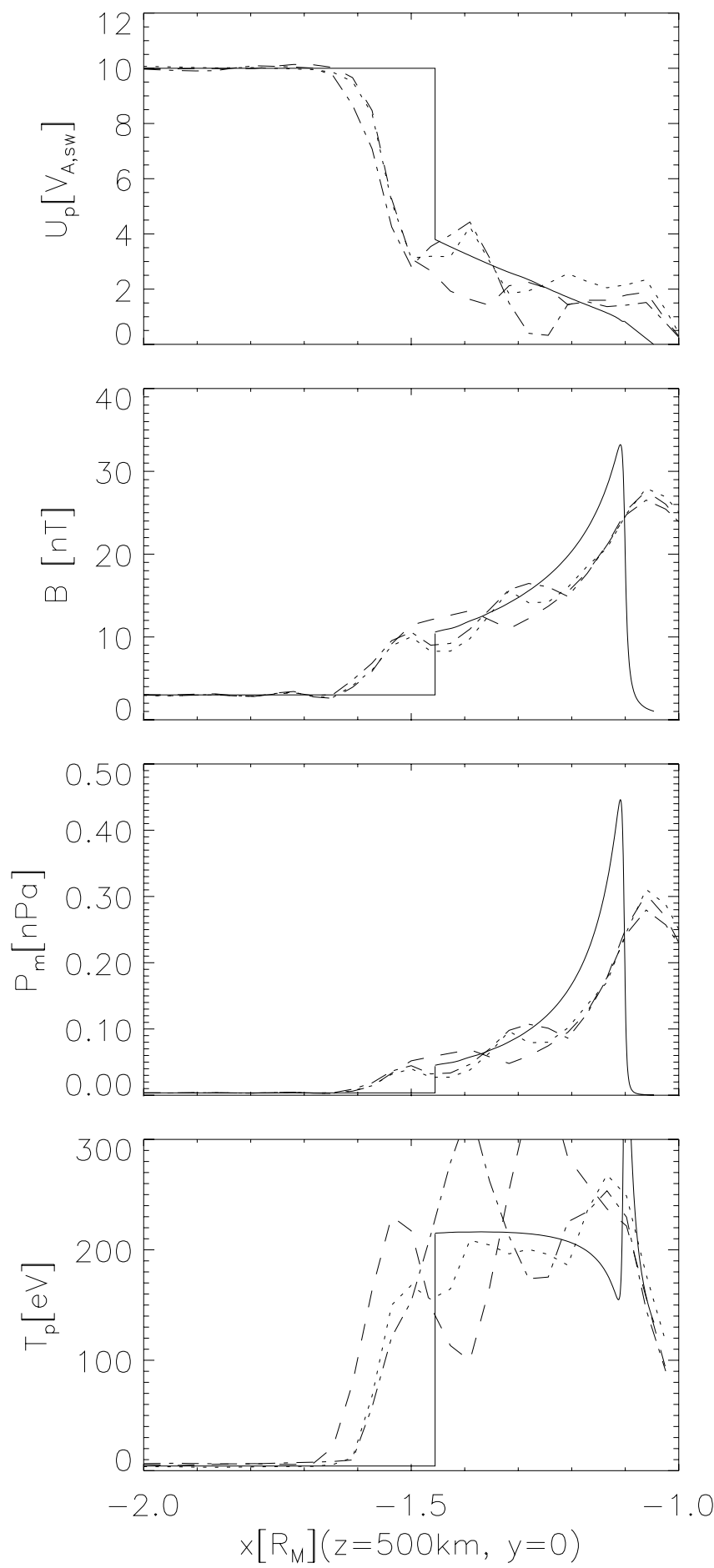

Fig. 3b. Comparison of MHD and hybrid magnetosheath profiles along the $x$ direction for $z=500 \mathrm{~km}, y=0$.

stand-off distances was found to be $\sim 1.7 R_{M}$ (Vignes et al., 2000). The corresponding ratio of the stand-off distance to the effective obstacle radius is equal to $\sim 1.31$. In the present MHD simulation, the ratio of the subsolar stand-off distance and the obstacle radius is equal to 1.32 , which is rather close to the empirical value.
The electric field has large fluctuations but its average value decreases towards the obstacle. At the stagnation point where the MHD electric field vanishes, the hybrid electric field does not equal zero because of magnetic field diffusion.

In both models, the proton velocity given in units of the solar wind Alfvén speed decreases at the shock front. In the 


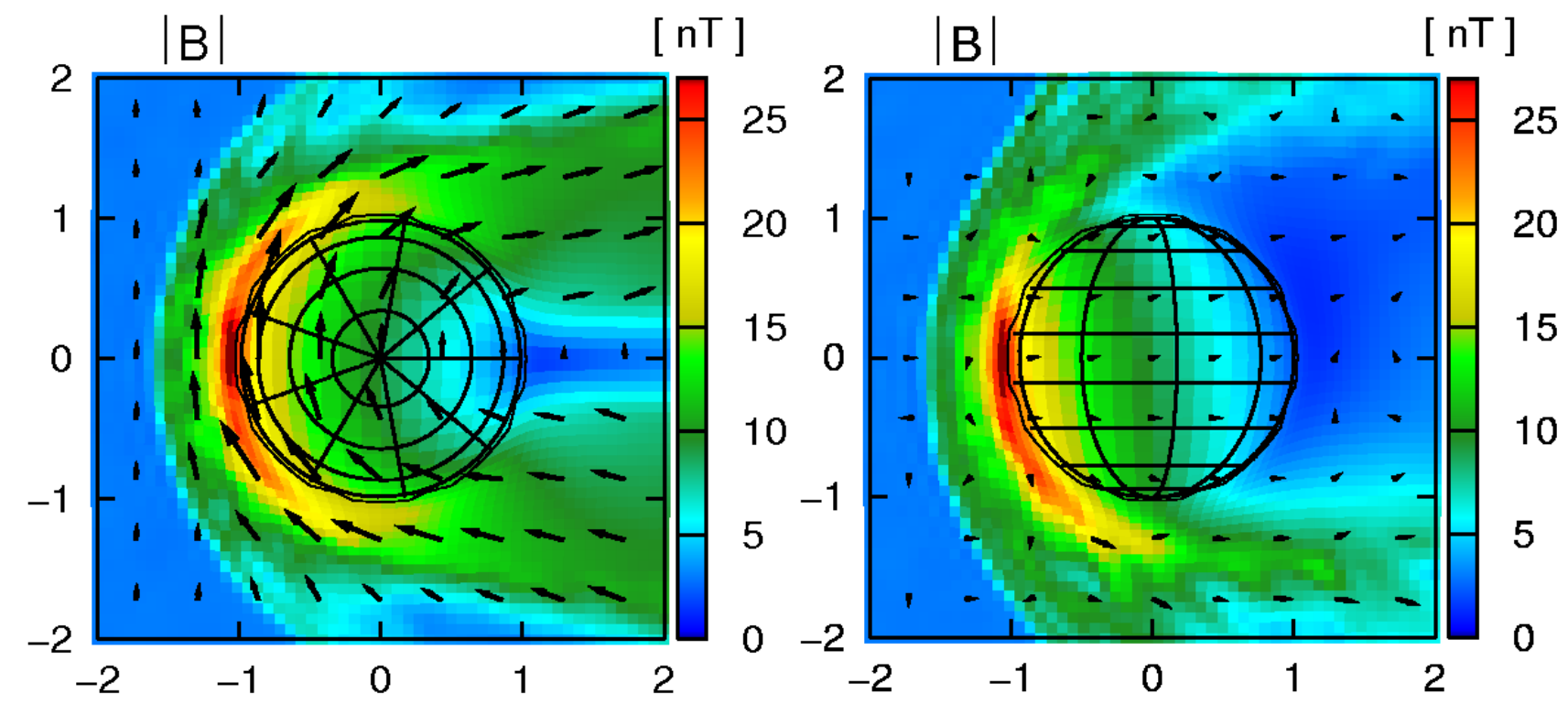

Fig. 4. Hybrid simulation: Distribution of the magnetic field strength in the $x y$ plane (left) and $x z$ plane (right).

hybrid simulations, there is a correlation between the proton velocity and electric field variations which is clearly indicated by Figs. 2, 3a. Maximal and minimal values of the oscillating electric field and proton velocity are corresponding to each other. In spite of the fluctuations, the average velocity in the hybrid simulations agrees with that of the MHD model. However, at the ICB, the proton's velocity in the hybrid model does not vanish. This might be related to a proton drift through the ICB which is caused by the nonzero electric field at the boundary. An additional possible reason is that a few energetic protons can cross the ICB.

A quite good agreement between the models appears for the magnetic field profiles along most of the magnetosheath. The magnetic field behavior in the subsolar magnetosheath may be described as follows: the magnetic field increases monotonically from the bow shock towards the ICB. The scaling of the magnetic field maximum is $B \sim \sqrt{4 \pi \rho_{s w}} u_{s w}$. The factor of the total magnetic field enhancement is of the order of the solar wind Alfvén Mach number $B_{\max } / B_{s w} \sim M_{A}$. In the MHD simulation, the magnetic field increases by a factor of 12 for $M_{A}=10$. In the hybrid simulation the magnetic field maximum is a bit less because of magnetic diffusion into the ionosphere. The magnetic pressure has a steep rise towards the obstacle; it increases by a factor of 2 on a length scale $0.1 R_{m}=340 \mathrm{~km}$. The growth of the magnetic pressure is accompanied by a simultaneous drop in the plasma pressure. The maximum of the magnetic pressure is approximately equal to the solar wind dynamic pressure. The position of the magnetic field maximum in the simulations coincides with the total density pile-up boundary.
The subsolar magnetosheath profiles of the magnetic field and electron density shown in Fig. 2 are rather similar to those obtained from Phobos-2 measurements (see Fig. 1a) and also from bi-ion fluid simulations by Sauer and Dubinin (2000).

In the hybrid simulations, the proton temperature has large oscillations appearing just after the shock. However, the average value seems to be rather close to that obtained from the Rankine-Hugoniot equations.

In the ideal MHD simulations, the magnetic field has a very sharp decrease from the ICB towards the planet. The strong decrease in the magnetic pressure is compensated by a corresponding increase in the plasma pressure. This structure can be unstable with respect to an interchange instability, which leads to the penetration of magnetic tubes into the ionosphere. In the hybrid simulations, the magnetic field penetrates inside the ionosphere because of magnetic diffusion effects.

Figures 4 and 5 present the distributions of the magnetic field strength and proton density in the $x y$ and $x z$ planes which are obtained in the hybrid simulation. In the $x y$ plane, the distributions of the magnetic field and plasma density are rather symmetrical, whereas in the $x z$ plane one can see a strong asymmetry in the direction of the solar wind convection electric field, related to finite gyro-radius effects. Such asymmetries were also reproduced in other hybrid simulations (e.g. Shimazu, 2001; Kallio and Janhunen, 2002).

The magnetosheath magnetic barrier is indicated in Fig. 4 by the red color in the dayside magnetosheath region. Its size along the y-direction is about $2 R_{M}$. This is similar to that obtained in the MHD simulation (see Fig. 6). A particular feature of the hybrid simulation is a penetration of the 


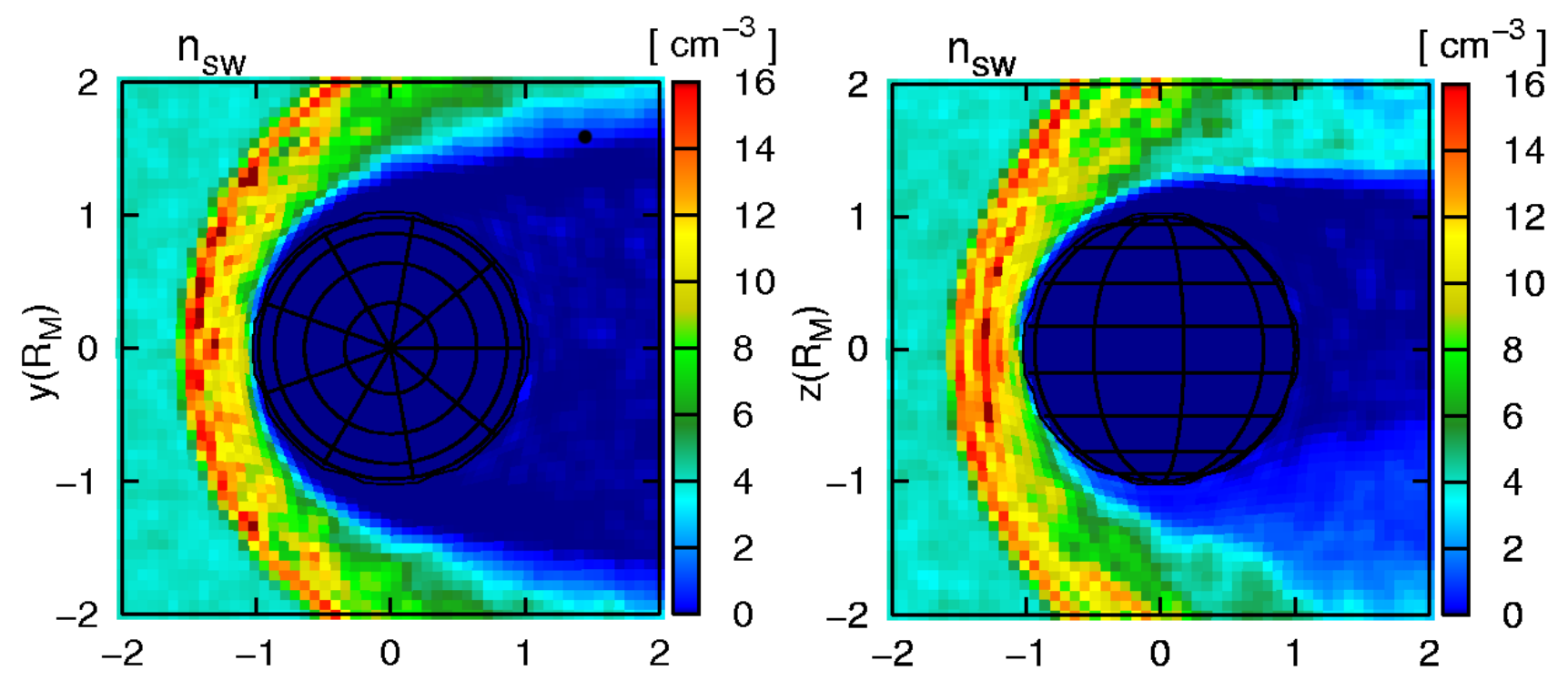

Fig. 5. Hybrid simulation: Distribution of the proton density in the $x y$ plane (left) and $x z$ plane (right).

solar wind magnetic field into the ionosphere because of the magnetic diffusion effects.

The distribution of the solar wind protons shown in Fig. 5 is rather patchy. At the subsolar bow shock, the proton density is of the same order of magnitude as that in the MHD simulation. But in the middle part of the magnetosheath, the hybrid proton density is somewhat lower than that calculated in the MHD model. In the vicinity of the ICB, the proton density has a very sharp decrease similar to the MHD result. There is a distinct slightly expanding boundary where the proton density drops to zero.

Figures 6, 7, 8 show the MHD model distributions of the magnetic field strength, proton density and plasma pressure in the magnetosheath in two planes, $x y$ and $x z$, which are coplanar and perpendicular to the IMF, respectively. The undisturbed IMF lines are shown in the $x y$ plane as straight lines along the y-direction. Contrary to the hybrid model, the distributions of the magnetic field and plasma parameters are symmetrical in both planes. The coordinates $\mathrm{x}, \mathrm{y}$ are given in units of the planetary radius. In Fig. 6 one can see explicitly the enhanced magnetic field strength in the magnetic barrier (red layer) adjacent to the dayside magnetopause. In the magnetosheath, the behavior of the plasma pressure is opposite to that of the magnetic field strength. The proton density has a systematic decrease from the bow shock towards the obstacle.

Good agreement between the MHD and hybrid magnetic field profiles in the magnetosheath of Mars can be interpreted on the basis of the following physical reasons: in the ideal MHD model, the physical reason for the magnetic pile-up is a stretching of the magnetic field lines which are frozen-in to the magnetosheath plasma flow. In the hybrid model, the magnetic field is frozen-in only to electrons, but not to protons, but effectively, it behaves as if it were frozen-in to the whole plasma in most of the dayside magnetosheath. This means that the relative speed of the electrons and protons (electric current speed) is much smaller than the bulk speed of the protons. To check this condition we express the electron velocity using Eq. (19),

$\boldsymbol{u}_{e}=\boldsymbol{u}_{p}+\frac{n_{i}}{n}\left(\boldsymbol{u}_{i}-\boldsymbol{u}_{p}\right)-\frac{c}{4 \pi n e} \nabla \times \boldsymbol{B}$.

Eliminating the electron velocity from induction Eq. (21), we obtain

$$
\begin{aligned}
\frac{\partial \boldsymbol{B}}{\partial t}= & \left.\nabla \times\left(\boldsymbol{u}_{p} \times \boldsymbol{B}\right)+\nabla \times\left[\frac{n_{i}}{n}\left(\boldsymbol{u}_{i}-\boldsymbol{u}_{p}\right) \times \boldsymbol{B}\right)\right]- \\
& \nabla \times\left[\frac{1}{4 \pi e n}(\nabla \times \boldsymbol{B}) \times \boldsymbol{B}\right] .
\end{aligned}
$$

With dimensionless quantities normalized to the solar wind parameters and a length scale for the magnetic barrier $\Delta_{m}$, Eq. (23) yields

$$
\begin{aligned}
\frac{\partial \tilde{\boldsymbol{B}}}{\partial \tilde{t}}= & \left.\nabla \times\left(\tilde{\boldsymbol{u}}_{p} \times \tilde{\boldsymbol{B}}\right)+\nabla \times\left[\frac{n_{i}}{n}\left(\tilde{\boldsymbol{u}}_{i}-\tilde{\boldsymbol{u}}_{p}\right) \times \tilde{\boldsymbol{B}}\right)\right]- \\
& \frac{\delta_{p}}{M_{A} \Delta_{m}} \nabla \times\left[\frac{1}{\tilde{n}}(\nabla \times \tilde{\boldsymbol{B}}) \times \tilde{\boldsymbol{B}}\right],
\end{aligned}
$$

where $\delta_{p}$ is the inertial scale of the solar wind protons $\delta_{p}=c \sqrt{M_{p}} / \sqrt{4 \pi n_{s w}} e$. The second term can be neglected while the ratio of the heavy ion density to the total density is small. It becomes important only in the vicinity of the ICB. The third term in Eq. (24) has a small coefficient

$\xi=\frac{1}{M_{A}} \frac{\delta_{p}}{\Delta_{m}}$. 


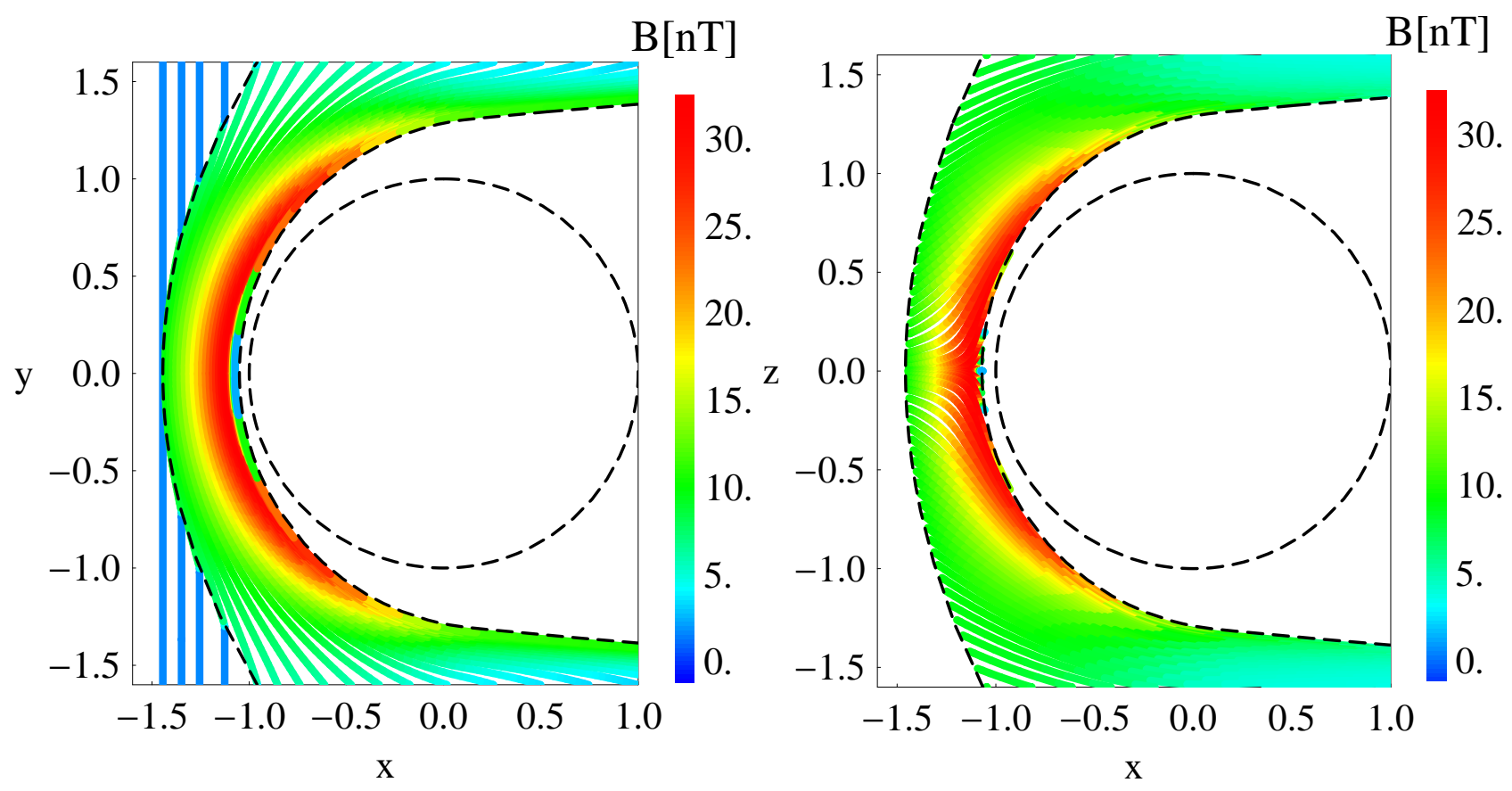

Fig. 6. MHD model: Distribution of the magnetic field strength in the $x y$ plane (left) and $x z$ plane (right).

For the input solar wind parameters (11) we find $M_{A}=10$, $\delta_{p}=114 \mathrm{~km}$. Taking the scale $\Delta_{m} \sim 0.1 R_{M}$ from the magnetosheath profiles presented in the figures, we estimate the coefficient $\xi=0.034$. Therefore, for most of the dayside magnetosheath, we can neglect the last term in the induction Eq. (24). This means that the magnetic field can be considered to be frozen-in to the plasma, as it is assumed in the ideal MHD. However, the last term becomes important in the vicinity of the ICB, where the magnetic field has a large gradient.

In the MHD simulation, the assumed ratio of specific heat $(\kappa=5 / 3)$ is relevant to the solar wind protons and oxygen atomic ions. In the hybrid simulations, the protons and ions are treated kinetically, but electrons are considered to be a polytropic gas $\left(p_{e} \sim n_{e}^{\kappa}\right)$ with the polytropic index $\kappa=2$ which is different from that used in the MHD calculations. However, this difference does not greatly affect the magnetosheath profiles, because the electron pressure is rather small compared to the proton pressure.

As distinguished from other MHD codes, the present MHD code based on the frozen-in coordinates does not have a numerical magnetic diffusion. Therefore, it describes an ideal MHD flow with frozen-in magnetic field lines. In this model, the magnetosheath plasma flow produces a stretching of the magnetic field lines, which results in the formation of the magnetic barrier in front of the ICB where the protons are stopped. In the ideal one-fluid MHD model, the interplanetary magnetic field is enhanced just before the proton stoppage boundary associated with the ICB, and the mag- netic field lines cannot penetrate inside the cavity. In a twofluid MHD and also in hybrid models, the magnetic field lines and protons are decoupled and thus the interplanetary magnetic field lines are allowed to penetrate through the proton stoppage boundary. However, numerical diffusion effects usually lead to smoothing of the magnetic field boundaries. The present hybrid model has sufficient resolution to produce a rather distinct boundary of the proton cavity which coincides with the magnetic field maximum at the subsolar region. However, the magnetic field variation through the cavity boundary is rather smooth.

\section{On magnetic field diffusion into the ionosphere}

In this section we discuss physical reasons for the magnetic field diffusion into the ionosphere which is seen in the hybrid simulations.

The cold, heavy ions inside the ionosphere are assumed to obey the equations

$$
\begin{aligned}
M_{i}\left(\frac{\partial \boldsymbol{u}_{i}}{\partial t}+\boldsymbol{u}_{i} \nabla \boldsymbol{u}_{i}\right)= & e\left(\boldsymbol{E}+\frac{1}{c} \boldsymbol{u}_{i} \times \boldsymbol{B}\right) \\
& -M_{i} K_{D} N_{0} \boldsymbol{u}_{i} .
\end{aligned}
$$

The last term increasing towards the planet is that of the interaction with neutral gas, which is assumed to be at rest. 


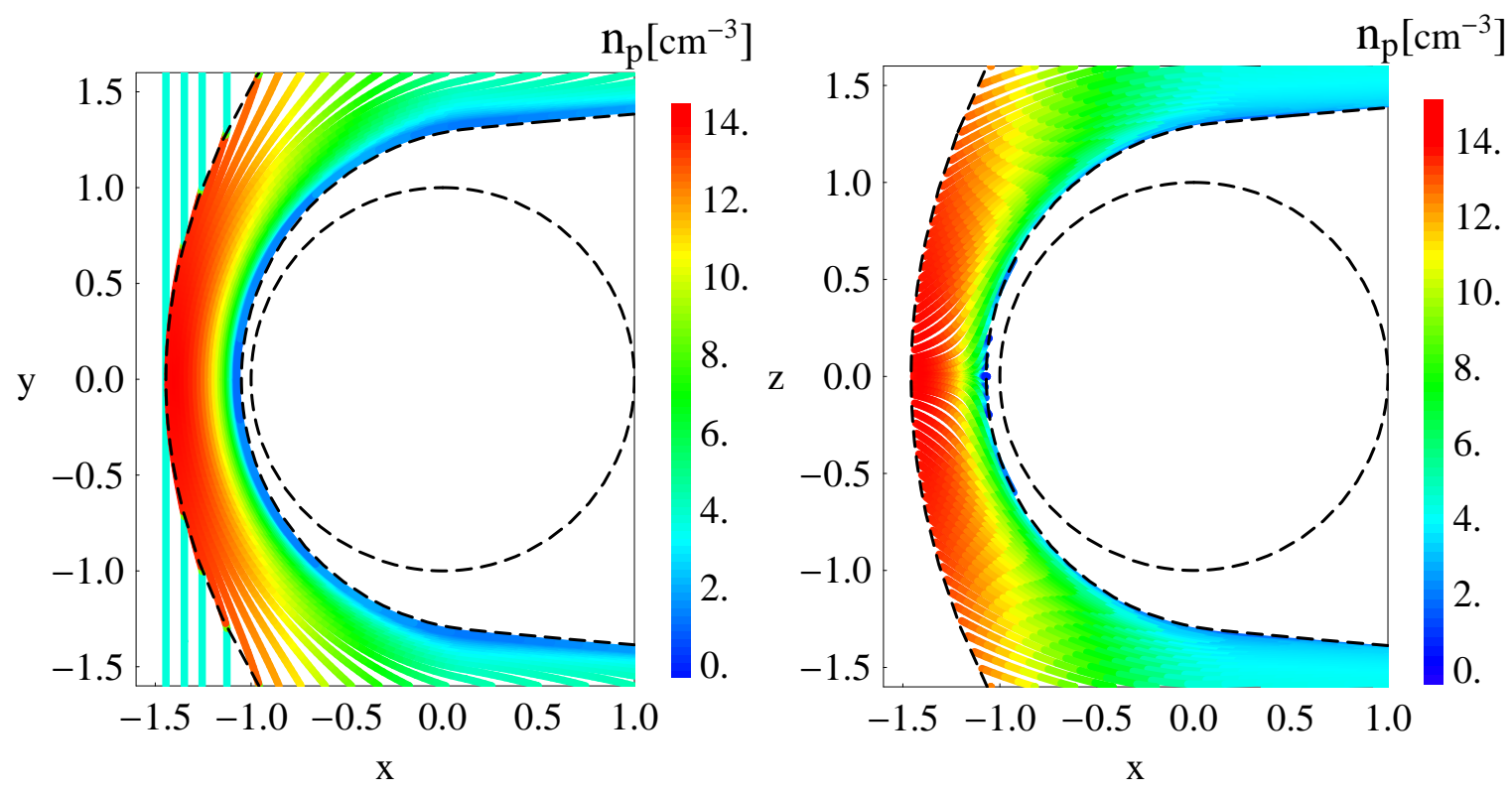

Fig. 7. MHD model: Distribution of the proton density in $x y$ plane (left) and $x z$ plane (right).

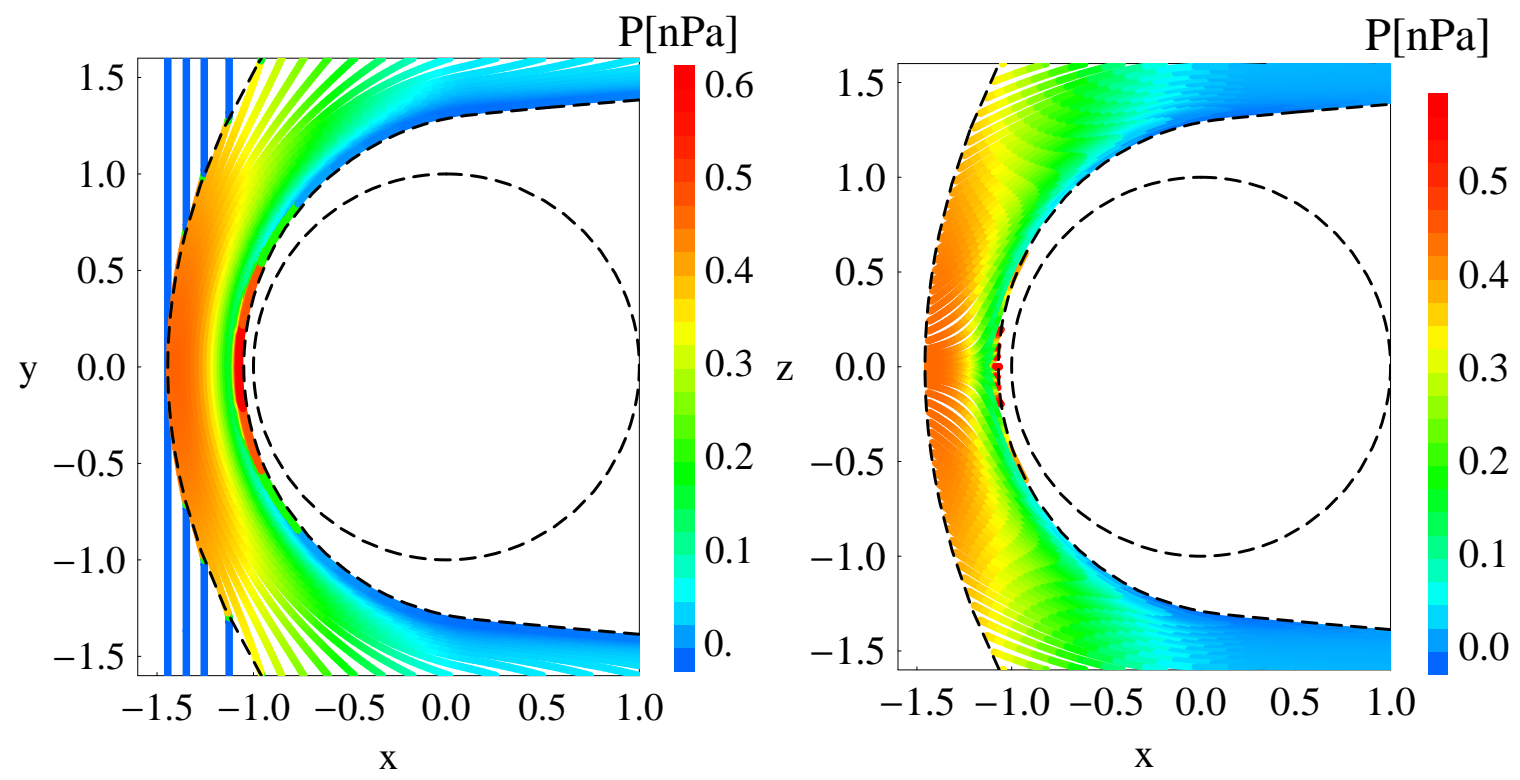

Fig. 8. MHD model: Distribution of the plasma pressure in $x y$ plane (left) and in $x z$ plane (right).

We introduce the normalized physical quantities,

$\boldsymbol{B}=\tilde{\boldsymbol{B}} B^{*}, \quad \boldsymbol{u}_{i}=\tilde{\boldsymbol{u}}_{i} \frac{c E^{*}}{B^{*}}, \quad t=\tilde{t} \frac{R_{M} B^{*}}{c E^{*}}$,

$\boldsymbol{r}=\tilde{\boldsymbol{r}} R_{M}, \quad \boldsymbol{E}=\tilde{\boldsymbol{E}} E^{*}$,

and rewrite Eq. (26) in a dimensionless form

$\mu\left(\frac{\partial \tilde{\boldsymbol{u}}_{i}}{\partial t}+\tilde{\boldsymbol{u}}_{i} \nabla \tilde{\boldsymbol{u}}_{i}\right)=\eta\left(\boldsymbol{E}+\tilde{\boldsymbol{u}}_{i} \times \tilde{\boldsymbol{B}}\right)-\tilde{\boldsymbol{u}}_{i}$, where

$\mu=\frac{c E^{*}}{R_{M} K_{D} N_{0} B^{*}}, \quad \eta=\frac{e B^{*}}{M_{i} c K_{D} N_{0}}$.

Coefficients $\mu$ and $\eta$ are inversely proportional to the neutral density $N_{0}$, and thus they decrease very strongly towards the Martian surface. Figure 9 shows variations of coefficients $\mu$ 
and $\eta$ along the $x$ axis for the normalization parameters

$B^{*}=10 \mathrm{nT}, \quad E^{*}=0.1 \mathrm{~V} / \mathrm{km}$.

These normalization parameters are of the order of the characteristic values of the magnetic and electric field as obtained in the hybrid simulation in the ionospheric region. One can see in Fig. 9 that coefficient $\mu$ is rather small until the altitude of $270 \mathrm{~km}$.

Neglecting the term proportional to the small coefficient $\mu$, we obtain the linear algebraic equation for the ion velocity

$\eta\left(\tilde{\boldsymbol{E}}+\tilde{\boldsymbol{u}}_{i} \times \tilde{\boldsymbol{B}}\right)-\tilde{\boldsymbol{u}}_{i}=0$.

Assuming the magnetic field along the $y$ direction, we find the ion velocity components as linear functions of the electric field components,

$\tilde{u}_{i, x}=\frac{\eta}{1+\eta^{2} \tilde{B}^{2}} \tilde{E}_{x}-\frac{\eta^{2} \tilde{B}}{1+\eta^{2} \tilde{B}^{2}} \tilde{E}_{z}$,

$\tilde{u}_{i, y}=\eta \tilde{E}_{y}$

$\tilde{u}_{i, z}=\frac{\eta}{1+\eta^{2} \tilde{B}^{2}} \tilde{E}_{z}+\frac{\eta^{2} \tilde{B}}{1+\eta^{2} \tilde{B}^{2}} \tilde{E}_{x}$.

Using Eq. (32) for the electric current of the ions $\boldsymbol{j}_{i}=e n V^{*} \tilde{\boldsymbol{u}}_{i}$, one can obtain Ohm's law for the ions with a tensor conductivity,

$j_{i}=\Sigma \boldsymbol{E}$

The nondiagonal components of the conductivity tensor become of the order of the diagonal ones at altitudes exceeding $225 \mathrm{~km}$. Below, the conductivity tensor can be considered as a diagonal $\Sigma=\sigma \mathbf{I}$, where $\sigma$ is a scalar conductivity,

$\sigma=\frac{n_{i} e^{2}}{M_{i} K_{D} N_{0}}$

At the subsolar region, considering the electric field to be along $z$, we have the equation

$E_{z}=\sigma j_{z}$

In the subsolar region, the length scale of the magnetic field variation along the $x$-direction is much smaller than the length scales along the $y$ - and z-directions (see Fig. 4). Therefore, we assume the derivative along the $\mathrm{x}$-axis to be much larger than those along the $y$ - and $z$-directions. From Eq. (35) and the Maxwellian equations, we obtain the magnetic diffusion equation

$\frac{\partial B_{y}}{\partial t}=\frac{\partial}{\partial x}\left(v_{m} \frac{\partial B_{y}}{\partial x}\right)$,

where $v_{m}$ is the magnetic diffusion coefficient,

$v_{m}=\frac{c^{2}}{4 \pi \sigma}$.

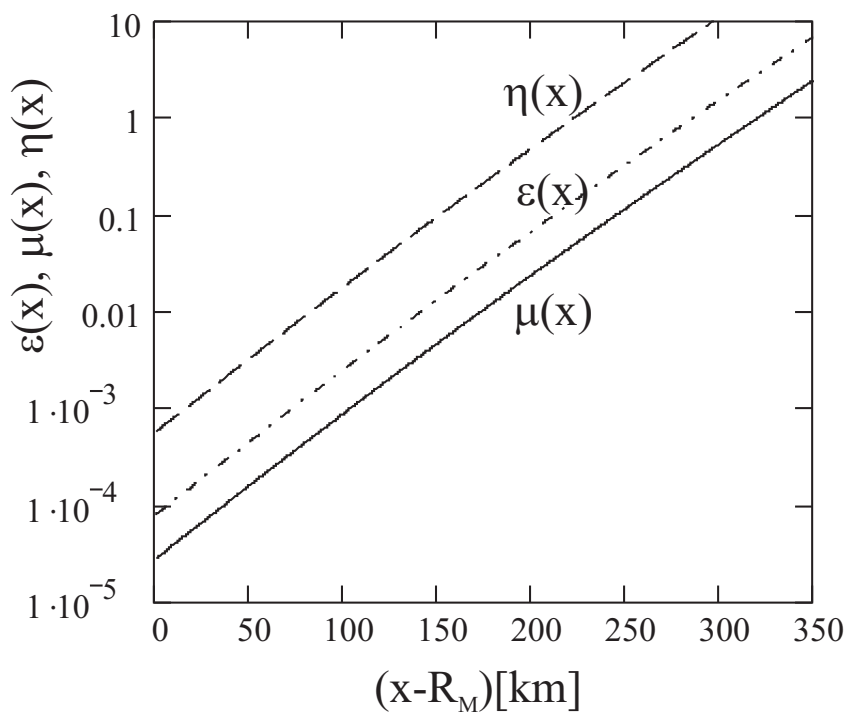

Fig. 9. Altitude dependence of the dimensionless parameters: dash, dash-and-dot, and solid lines are corresponding to $\eta, \epsilon$, and $\mu$, respectively.

The time scale of the magnetic field diffusion is

$\tau_{d}=\frac{1}{c^{2}} 4 \pi \sigma \Delta_{s}$,

where $\Delta_{s}$ is the subsolar altitude of the ICB. We introduce a dimensionless parameter $\epsilon$ that is a ratio of the diffusion time scale to the simulation time $t^{*} \sim 2500 \mathrm{~s}$,

$\epsilon(x)=\frac{4 \pi e^{2} \Delta_{s}^{2} n_{i}}{c^{2} t^{*} k_{D} N_{0} M_{i}}$.

Small values of this parameter mean that the simulation time exceeds the magnetic diffusion time, and thus the magnetic field can penetrate into the ionosphere until the Martian surface during the simulation run. Figure 9 shows the altitude dependence of the diffusion time parameter $\epsilon(x)$. This parameter becomes less than 1 at the altitude $\leq 285 \mathrm{~km}$, where the magnetic field diffusion becomes important due to collisions with neutrals. Above this altitude, a magnetic diffusion might have only numerical reasons.

\section{Conclusions}

The solar wind interaction with Mars is considered on a basis of the ideal MHD and hybrid models. The magnetosheath profiles of the magnetic field and plasma parameters, calculated in both models, are compared.

The stand-off distance of the bow shock in the hybrid model is larger than that in the MHD model. This can be explained by two reasons: the first one is the finite width of the shock front that is of the order of the Larmour radius. The second reason can be related to the additional penetration of 
the heavy ions dragged by the electric field from the ionosphere in the hybrid simulation. Acceleration of these ions leads to a decrease in the average speed of the protons and a corresponding increase in the magnetosheath thickness.

However, good agreement between the models is found for the magnetic field and plasma parameters just after the bow shock. This means that in spite of the oscillations, the hybrid model can reproduce a thermalization of plasma at the bow shock and satisfy the Rankine-Hugoniot conditions based on conservation of mass, momentum and energy.

A particular good agreement between the MHD and hybrid models is obtained for the subsolar magnetic field profiles between the bow shock and the ICB. This allows us to suggest that the physical mechanism of the magnetosheath magnetic barrier described by the ideal MHD model is also valid for relatively small bodies like Mars. In the ideal MHD model, the physical reason for the magnetosheath magnetic barrier is a stretching of the magnetic field lines which are frozen-in to the plasma in the magnetosheath flow. In the hybrid model, the magnetic field generally is frozen-in to the electrons, but not to the protons, but effectively, it behaves as if it were frozen-in to the whole plasma in the part of the dayside magnetosheath where the ionospheric ions are a minor component. This means that the relative speed of the electrons and protons (electric current speed) is much smaller than the bulk speed of the protons. This condition is not fulfilled in the vicinity of the ICB, where the electric current speed becomes comparable to the bulk velocity, and the magnetic field is frozen-in to the electrons only.

We have focused our analysis only on the magnetosheath region above the proton cavity, because the enhanced magnetic field inside the cavity cannot be described by the ideal MHD model. The induced magnetosphere is reproduced by the hybrid model, but the magnetic field distribution and the MPB structure are greatly smoothed by the numerical diffusion. Therefore, the hybrid model does not predict a sudden jump in the magnetic field strength at the MPB, as it is identified in the observations (Nagy et al., 2004).

The physical reasons for the magnetic field penetration into the ionosphere are analyzed. The magnetic field diffusion due to collisions with the neutral particles is found to be essential at the altitude of $55 \mathrm{~km}$ below the ICB. For the conditions of the hybrid simulations, the magnetic field penetrates from ICB until the diffusion zone because the electron pressure itself is not sufficient to support the magnetic boundary.

Acknowledgements. This work was supported by grant Y 436RUS17/121/05 and Mo 539/13-2 from the "Deutsche Forschungsgemeinschaft". Additional support is due to grant 04-05-64088 from the Russian Foundation of Basic Research, by Programs 2.17 and 16.3 of the Russian Academy of Sciences, by project P17100-N08 from the Austrian "Fonds zur Förderung der wissenschaftlichen Forschung", and also by project I.2/04 from "Österreichischer Austauschdienst".
Topical Editor I. A. Daglis thanks two referees for their help in evaluating this paper.

\section{References}

Biernat, H. K., Erkaev, N. V., and Farrugia, C. J.: Aspects of MHD flow about Venus, J. Geophys. Res., 104, 12 617-12 626, 1999.

Biernat, H. K., Erkaev, N. V., and Farrugia, C. J.: MHD effects in the Venus magnetosheath including mass loading, Adv. Space Res., 28, 833-839, 2001.

Breus, T. K., Krymskii, A. M., Lundin, R., Dubinin, E. M., Luhmann, J. G., Yeroshenko, Y. G., Barabash, S. V., Mitnitskii, V. Y., Pissarenko, N. F., and Styashkin, V. A.: The solar wind interaction with Mars: consideration of Phobos-2 mission observations of an ion composition boundary on the dayside, J. Geophys. Res., 96, 11 165-11 174, 1991.

Brecht, S. H., Ferrante, J. R., and Luhmann, J. G.: Threedimensional simulations of the solar wind interaction with Mars, J. Geophys. Res., 98, 1345-1357, 1993.

Brecht, S. H.: Hybrid simulations of the magnetic topology of Mars, J. Geophys. Res., 102, 4743-4750, 1997.

Bößwetter, A, Bagdonat, T., Motschmann, U., and Sauer, K.: Plasma boundaries at Mars: a 3-D simulation study, Ann. Geophys., 22, 4363-4379, 2004, http://www.ann-geophys.net/22/4363/2004/.

Chamberlain, J. W. and Hunten, D. M.: Theory of planetary atmospheres, Academic Press, New York, 1987.

Chen, R. H., Cravens, T. E., and Nagy, A. F.: The Martian ionosphere in light of the Viking observations, J. Geophys. Res., 83, 3871-3876, 1978.

Erkaev, N. V., Farrugia, C. J., and Biernat, H. K.: Effects on the Jovian magnetosheath arising from solar wind flow around nonaxial bodies, J. Geophys. Res., 101, 10 665-10 672, 1996.

Erkaev, N. V., Farrugia, C. J., and Biernat, H. K.: Comparison of gasdynamics and MHD predictions for magnetosheath flow, in: NATO ASI Series, Polar Cap Boundary Phenomena, edited by: Moen, J., Egeland, A., and Lockwood, M., Kluwer Academic Publisher, Dordrecht, The Netherlands, 509, p. 27-40, 1998.

Erkaev, N. V., Farrugia, C. J., and Biernat, H. K.: Threedimensional, one-fluid, ideal MHD model of magnetosheath flow with anisotropic pressure, J. Geophys. Res., 104, 6877-6888, 1999.

Erkaev, N. V., Farrugia, C. J., and Biernat, H. K.: The role of the magnetic barrier in the Solar wind-magnetosphere interaction, Planet. Space Sci., 51, 745-755, 2003.

Hanson, W. B. and Mantas, G. P.: Viking electron temperature measurements: Evidence for a magnetic field in the Martian ionosphere, J. Geophys. Res., 93, 7538-7544, 1988.

Israelevich, P. L., Gombosi, T. I., Ershkovich, A. I., DeZeeuw, D. L., Neubauer, F. M., and Powell, K. G.: The induced magnetosphere of comet Halley, 4, Comparison of in situ observations and numerical simulations, J. Geophys. Res., 104, $28309-$ 28319, 1999.

Kallio, E. and Luhmann, J. G.: Charge exchange near Mars: The solar wind absorption and energetic neutral atom production, J. Geophys. Res., 102, 22 183-22 197, 1997.

Kallio, E. and Janhunen, P.: Atmospheric effects of proton precipitation in the Martian atmosphere and its connection to the Marssolar wind interaction, J. Geophys. Res., 106, 5617-5634, 2001. 
Kallio, E. and Janhunen, P.: Ion escape in a quasi-neutral hybrid model, J. Geophys. Res., 107, 1035, doi:10.1029/2001J000090, 2002.

Kotova, G., Verigin, M., Remizov, A., Rosenbauer, H., Livi, S., Szegö, K., Tatrallyay, M., Slavin, J., Lemaire, J., Schwingenschuh, K., and Zhang, T. L.: Study of the solar wind deceleration upstream of the Martian terminator bow shock, J. Geophys. Res., 102, 2165-2173, 1997.

Liu, Y., Nagy, A. F., Gombosi, T. I., DeZeeuw, D. L., and Powell, K. G.: The solar wind interaction with Mars: results of threedimensional threespecies MHD studies, Adv. Space Res., 27, 1837-1846, 2001.

Ma, Y., Nagy, A. F., Sokolov, I. V., and Hansen, K. C.: Threedimensional, multispecies, high spatial resolution MHD studies of the solar wind interaction with Mars, J. Geophys. Res., 109, A07211, doi:10.1029/2003JA010367, 2004.

Nagy, A. F., Winterhalter, D., Sauer, K., Cravens, T. E., Brecht, S., Mazelle, C., Crider, D., Kallio, E., Zakharov, A., Dubinin, E., Verigin, M., Kotova, G., Axford, W. I., Bertucci, C., and Trotignon, J. G.: The plasma environment of Mars, Space Sci. Rev., 111, 33-114, 2004.

Petrinec, S. M. and Russell, C. T.: Hydrodynamic and MHD equations across the bow shock and along the surfaces of planetary obstacles, Space Sci. Rev., 79, 757-791, 1997.

Pudovkin, M. I. and Semenov, V. S.: Stationary frozen-in coordinate system, Ann. Geophys., 33, 429-433, 1977, http://www.ann-geophys.net/33/429/1977/.

Riedler, W., Schwingenschuh, K., Lichtenegger, H., Möhlmann, D., Rustenbach, J., Weroshenko, Y., Achache, J., Slavin, J., Luhmann, J. G., and Russell, C. T.: Interaction of the solar wind with the planet Mars: Phobos- 2 magnetic field observations, Planet. Space Sci., 39, 75-81, 1991.
Rosenbauer, H., Shutte, N., Apathy, I., Galeev, A., Gringauz, K., Grünwaldt, H., Hemmerich, P., Jockers, K., Kiraly, P., Kotova, G., Livi, S., Marsch, E., Richter, A., Riedler, W., Remizov, T., Schwenn, R., Schwingenschuh, K., Steller, M., Szegö, K., Verigin, M., and Witte, M.: Ions of Martian origin and plasma sheet in the Martian magnetoshere/initial results of the TAUS experiment, Nature, 341, 612-614, 1989.

Sauer, K., Roatsch, Th., Motschmann, U., Möhlmann, D., Schwingenschuh K., and Riedler, W.: Plasma boundaries at Mars discovered by the Phobos 2 magnetometers, Ann. Geophys., 8, 661670, 1990,

http://www.ann-geophys.net/8/661/1990/.

Sauer, K., Bogdanov, A., and Baumgaertel, K.: Evidence of an ion composition boundary (protonopause) in bi-ion fluid simulations of solar wind mass loading, Geophys. Res. Lett., 21, 2255-2258, 1994.

Sauer, K. and Dubinin, E.: The nature of the Martian .obstacle boundary., Adv. Space Res., 26, 1633-1637, 2000.

Shimazu, H.: Three-dimensional hybrid simulation of solar wind interaction with unmagnetized planets, J. Geophys. Res., 106, 8333-8342, 2001.

Spreiter, J. R. and Stahara, S. S.: A new predictive model for determining solar wind - terrestrial planet interactions, J. Geophys. Res., 85, 6769-6777, 1980.

Zwan, B. J. and Wolf, R. A.: Depletion of solar wind plasma near a planetary boundary, J. Geophys. Res., 81, 1636-1648, 1976.

Vignes, D., Mazelle, C., Rme, H., Acuna, M. H., Connerney, J. E. P., Lin, R. P., Mitchell, D. L., Cloutier, P., Crider, D. H., and Ness, N. F.: The solar wind interaction with Mars: locations and shapes of the bow shock and the observations of the MAG/ER experiment onboard Mars Global Surveyor, Geophys. Res. Lett., 27, 49-52, 2000. 